\title{
An MILP Approach for Short-Term Hydro Scheduling and Unit Commitment With Head-Dependent Reservoir
}

\author{
Alberto Borghetti, Senior Member, IEEE, Claudia D'Ambrosio, Andrea Lodi, and Silvano Martello
}

\begin{abstract}
The paper deals with a unit commitment problem of a generation company whose aim is to find the optimal scheduling of a multiunit pump-storage hydro power station, for a short term period in which the electricity prices are forecasted. The problem has a mixed-integer nonlinear structure, which makes very hard to handle the corresponding mathematical models. However, modern mixed-integer linear programming (MILP) software tools have reached a high efficiency, both in terms of solution accuracy and computing time. Hence we introduce MILP models of increasing complexity, which allow to accurately represent most of the hydroelectric system characteristics, and turn out to be computationally solvable. In particular we present a model that takes into account the head effects on power production through an enhanced linearization technique, and turns out to be more general and efficient than those available in the literature. The practical behavior of the models is analyzed through computational experiments on real-world data.
\end{abstract}

Index Terms-Hydro power plants operation, hydro reservoir management, mixed-integer linear programming, unit commitment.

\section{NOMENCLATURE:}

Sets:

$T=\{1, \ldots, \bar{t}\} \quad$ Set of time periods considered.

$J=\{1, \ldots, \bar{n}\} \quad$ Set of turbine-pump units.

\section{Parameters:}

$I_{t} \quad$ Predicted water inflow in period $t(t \in T)$ $\left[\mathrm{m}^{3} / \mathrm{s}\right]$.

$\Pi_{t} \quad$ Unit price of the power generated/consumed in period $t(t \in T)[€ / \mathrm{MWh}]$.

$\Delta t \quad$ Period duration $[\mathrm{h}]$.

$C_{j} \quad$ Startup cost of unit $j$ as a turbine $(j \in J)[€]$.

$D_{j} \quad$ Startup cost of unit $j$ as a pump $(j \in J)[€]$.

Manuscript received November 28, 2007; revised February 18, 2008. This work was supported in part by the Ministero dell'Università e della Ricerca under Project PRIN 2005 and in part by the Università di Bologna under Project Decisopelet 2006. Paper no. TPWRS-00900-2007.

A. Borghetti is with the Department of Electrical Engineering, University of Bologna, 40136 Bologna, Italy (e-mail: alberto.borghetti@unibo.it).

C. D'Ambrosio, A. Lodi, and S. Martello are with the Department of Electronics, Computer Sciences, and Systems, University of Bologna, 40136 Bologna, Italy (e-mail: c.dambrosio@unibo.it; andrea.lodi@unibo.it; silvano.martello@unibo.it).

Digital Object Identifier 10.1109/TPWRS.2008.926704
$\underline{Q}_{j}, \bar{Q}_{j} \quad$ Min and max flow value in turbine $j(j \in J)$ (when the turbine is on) $\left[\mathrm{m}^{3} / \mathrm{s}\right]$.

$\bar{P}_{j} \quad$ Max power produced by turbine $j(j \in J)$ [MW].

$\Delta q^{-}, \Delta q^{+} \quad$ Max ramp down and ramp up $\left[\mathrm{m}^{3} / \mathrm{s}\right]$.

$V, \bar{V} \quad$ Min and max water volume in the basin $\left[\mathrm{m}^{3}\right]$.

$V_{0} \quad$ Water volume in the basin in period $0\left[\mathrm{~m}^{3}\right]$.

$V_{\bar{t}} \quad$ Target (final) water volume in the basin $\left[\mathrm{m}^{3}\right]$.

$\bar{S} \quad$ Max water spillage $\left[\mathrm{m}^{3} / \mathrm{s}\right]$.

$W_{j} \quad$ Water needed to start up turbine $j(j \in J)$ $\left[\mathrm{m}^{3} / \mathrm{s}\right]$.

$Y_{j} \quad$ Water needed to start up pump $j(j \in J)$ $\left[\mathrm{m}^{3} / \mathrm{s}\right]$.

$E_{j} \quad$ Energy needed to start up pump $j(j \in J)$ [MWh].

$Q_{j 0} \quad$ Flow in turbine $j$ in period $0(j \in J)\left[\mathrm{m}^{3} / \mathrm{s}\right]$.

$G_{j 0} \quad$ Status of turbine $j$ in period $0(j \in J)[1$ on, 0 off].

$U_{j 0} \quad$ Status of pump $j$ in period $0(j \in J)[1$ on, 0 off].

$Q_{j}^{-} \quad$ Flow pumped by pump $j\left(j \in J ; Q_{j}^{-}<0\right)$ $\left[\mathrm{m}^{3} / \mathrm{s}\right]$.

$P_{j}^{-} \quad$ Power consumed during pumping by pump $j$ $\left(j \in J ; P_{j}^{-}<0\right)[\mathrm{MW}]$.

$\underline{\Theta} \quad$ Min released water in each period $\left[\mathrm{m}^{3} / \mathrm{s}\right]$.

Variables:

$q_{j t} \quad$ Water flow in unit $j$ in period $t(j \in J, t \in T)$, with $q_{j 0}=Q_{j 0}\left[\mathrm{~m}^{3} / \mathrm{s}\right]$.

$v_{t} \quad$ Water volume in the basin in period $t(t \in T)$, with $v_{0}=V_{0}\left[\mathrm{~m}^{3}\right]$.

$p_{j t} \quad$ Power generated or consumed by unit $j$ in period $t$ $(j \in J, t \in T)[\mathrm{MW}]$.

$s_{t} \quad$ Spillage in period $t(t \in T)\left[\mathrm{m}^{3} / \mathrm{s}\right]$.

$w_{j t} \quad$ Shutdown phase of turbine $j$ in period $t(j \in J$, $t \in T$ ) [1 if it is shutdown, 0 otherwise]. 
$\widetilde{w}_{j t} \quad$ Startup phase of turbine $j$ in period $t(j \in J, t \in T)$ [ 1 if it is started up, 0 otherwise].

$g_{j t} \quad$ Status of turbine $j$ in period $t(j \in J, t \in T)$, with $g_{j 0}=G_{j 0}[1$ on, 0 off $]$.

$y_{j t} \quad$ Shutdown phase of pump $j$ in period $t(j \in J$, $t \in T$ ) [1 if it is shutdown, 0 otherwise].

$\widetilde{y}_{j t} \quad$ Startup phase of pump $j$ in period $t(j \in J, t \in T)$ [ 1 if it is started up, 0 otherwise].

$u_{j t} \quad$ Status of pump $j$ in period $t(j \in J, t \in T)$, with $u_{j 0}=U_{j 0}[1$ on, 0 off $]$.

Some additional parameters and variables, introduced to linearize the model, are defined in Section II.

\section{INTRODUCTION}

W E consider a price-taker generating company that wants to optimize the operation of a pump-storage multiunit hydro power station for a given time horizon, typically one day or one week. The problem is to determine the commitment and the power generation of the plant so as to maximize the revenue given by power selling. All the units of the plant are assumed to be fed by the same reservoir. We assume that inflows and prices are known as previously forecasted.

Several approaches have been proposed for the solution of this problem. For an exhaustive overview we refer the reader to the recent survey [1]. In [2] the problem was formulated as a simple linear programming (LP) model by neglecting costs and constraints relevant to startups and shutdowns. In [3] a nonlinear programming (NLP) model with some simplified assumption was introduced. Ad-hoc heuristics were proposed by several authors, such as [4] and [5]. In [6] a multistage looping optimization algorithm was proposed for the development of the optimal bidding strategies of an individual pumped-storage unit owner in a competitive electricity market. In [7] the large-scale mixed-integer NLP problem of determining the optimal scheduling of hydropower plants in a hydrothermal interconnected system is considered: the authors use Lagrangian relaxation decomposition strategies, and a sequential quadratic programming algorithm to solve nonlinear subproblems. Various mixed-integer linear programming (MILP) approaches have been presented in the literature: for example, [8] and [9] used the interior point method within a branch-and-bound algorithm, while [10]-[13] used the Ilog-Cplex [14] MILP solver under GAMS.

Although we limit the analysis to the case of a single reservoir, the problem is especially interesting because both of its practical relevance and of the difficulties induced by its nonlinear aspects, namely the relationship between the unit electrical power output and the corresponding water flow derived from the reservoir, particularly if the so called head effect, i.e., the influence on power production of the water level in the reservoir, has to be taken into account. We focus on the modeling of this nonlinear characteristic, and show how it can be efficiently and accurately dealt with by using MILP techniques. Indeed, the high efficiency of modern MILP software tools, both in terms of solution accuracy and computing time, encourage their use also in the solution of nonlinear problems.
The proposed MILP model allows one to accurately represent the main technical and operating characteristics of a pumpstorage multiunit hydro power plant, and turns out to be computationally solvable for a planning horizon of one week. For the general structure of the MILP model, we follow the one proposed in [13]. The differences mainly refer to the following aspects: 1) the proposed model takes into account some additional characteristics of the hydro units, such as ramp transition constraints and pump-storage operating mode; 2 ) we introduce a more sophisticated modeling of the head effect through a specialized approximation methodology (based on two dimensional considerations) for the relationship among power, volume and flow.

The paper is organized as follows. In Sections II-A and II-B we give the main components of the proposed model, with special emphasis on costs and constraints related to turbine and pump startups and to the linearization of the relationship between power and water flow. The most sophisticated version of the model, which allows a tight representation of the head effect, is presented in Section III. The model is then computationally evaluated in Section IV through experiments on real-world data. Instances where the pumps have prohibited zones are also evaluated. Conclusions and directions for future researches are finally given in Section V.

\section{MAthematicAl Model}

In our nomenclature all parameters are represented by upper capitals and all variables by lower capitals.

Preliminary observe that the parameters allow one to handle the pump start up in the two typical ways. If unit $j$ is started up as a pump by using another turbine of the power plant, then there is no energy consumption, but the relevant water spillage is taken into account. The opposite holds if pump $j$ is started up by using the energy provided by the external power network. In other words, the input has either $E_{j}=0$ (in the former case) or $Y_{j}=0$ (in the latter).

Note in addition that the first four variables are subject to the following obvious bounding constraints, for all $t \in T$ and $j \in J:$

$$
\begin{aligned}
& Q_{j}^{-} \leq q_{j t} \leq \bar{Q}_{j} \\
& \underline{V} \leq v_{t} \leq \bar{V} \\
& P_{j}^{-} \leq p_{j t} \leq \bar{P}_{j} \\
& 0 \leq s_{t} \leq \bar{S}
\end{aligned}
$$

and that, for any period $t$, the values of $q_{j t}$ and $p_{j t}$ depend on the three possible cases that can occur relative to turbine-pump unit $j$ :

TP10: if unit $j$ is generating power (i.e., $g_{j t}=1$ and $u_{j t}=$ $0)$ then both values are positive;

TP01: if unit $j$ is pumping water (i.e., $g_{j t}=0$ and $u_{j t}=1$ ) then both values are negative;

TP00: if unit $j$ is not operating (i.e., $g_{j t}=u_{j t}=0$ ) then both values are zero.

The model we propose aims at maximizing the sum, over all periods, of the profit given by power selling, minus the startup cost of each turbine-pump unit (if it occurs). Formally, this is represented by the linear objective function

$$
\max \sum_{j \in J} \sum_{t \in T}\left(\Delta t \Pi_{t} p_{j t}-C_{j} \widetilde{w}_{j t}-\left(D_{j}+\Pi_{t} E_{j}\right) \widetilde{y}_{j t}\right) \text {. }
$$


Note that the first term can take a negative value when the unit works as a pump.

The model can be logically subdivided into a set of "naturally" linear constraints and a set of nonlinear constraints, that are linearized in order to handle the model through MILP techniques.

Section II-A reports the set of "naturally" linear constraints. Sections II-B and III are devoted to the treatment of the nonlinear relationship between power production and water flow. In particular, we report in Section II-B an extension of the model of [13] and in Section III an enhanced version to better take into account the head effect.

\section{A. Linear Constraints}

The relationships among flow, volume and pumps/turbines status can be modeled through the following linear constraints:

$$
\begin{aligned}
& v_{\bar{t}}-V_{\bar{t}}=0 \\
& v_{t}-v_{t-1}- \\
& 3600 \Delta t\left(I_{t}-\sum_{j \in J} q_{j t}-s_{t}\right)=0, \quad \forall t \in T \\
& q_{j t}-\left(Q_{j}^{-} u_{j t}+\underline{Q}_{j} g_{j t}\right) \geq 0, \quad \forall j \in J, t \in T \\
& q_{j t}-\left(Q_{j}^{-} u_{j t}+\bar{Q}_{j} g_{j t}\right) \leq 0, \quad \forall j \in J, t \in T \\
& \sum_{j \in J}\left(q_{j t}-q_{j(t-1)}\right)+\Delta q^{-} \geq 0, \quad \forall t \in T \\
& \sum_{j \in J}\left(q_{j t}-q_{j(t-1)}\right)-\Delta q^{+} \leq 0, \quad \forall t \in T \\
& s_{t}-\sum_{j \in J}\left(W_{j} \widetilde{w}_{j t}+Y_{j} \widetilde{y}_{j t}\right) \geq 0, \quad \forall t \in T \\
& \sum_{j \in J} q_{j t}+s_{t}-\underline{\Theta} \geq 0, \quad \forall t \in T \\
& g_{j t}-g_{j(t-1)}-\left(\widetilde{w}_{j t}-w_{j t}\right)=0, \quad \forall j \in J, t \in T \\
& \widetilde{w}_{j t}+w_{j t} \leq 1, \quad \forall j \in J, t \in T \\
& u_{j t}-u_{j(t-1)}-\left(\widetilde{y}_{j t}-y_{j t}\right)=0, \quad \forall j \in J, t \in T \\
& \widetilde{y}_{j t}+y_{j t} \leq 1, \quad \forall j \in J, t \in T \\
& g_{j t}+u_{k t} \leq 1, \quad \forall j, k \in J, t \in T(14) \\
& \sum_{j \in J} u_{j t} \leq \bar{n}-1, \quad \forall t \in T \text {. }
\end{aligned}
$$

Constraint (2) sets the final water volume to the desired target value at the end of the considered time horizon $\bar{t}$. Constraints (3) impose the water conservation within two consecutive time periods. Constraints (4) and (5) establish lower and upper bounds on the flows in the turbines according to the three cases discussed above. Constraints (6) and (7) limit the flow variation within two consecutive periods. Constraints (8) impose the water spillage needed to startup a pump or a turbine. Constraints (9) establish a lower bound on the amount of water released in each period. Constraints (10) and (11) [resp. (12) and (13)] define the switch-on/switch-off rules of the turbines (resp. of the pumps). Constraints (14) impose that, if a turbine is on, no pump can be on and vice versa. Finally, constraints (15) are only introduced if the pumps startup method is to use the turbines: at least one pump is off because there are no turbines available to startup the last pump.

Note that an equivalent model could be obtained (see [15]) by eliminating the shutdown variables $w_{j t}$ and $y_{j t}$, and replacing constraints (10)-(13) with

$$
\begin{aligned}
& g_{j t}-g_{j(t-1)}-\widetilde{w}_{j t} \leq 0, \quad \forall j \in J, t \in T \\
& u_{j t}-u_{j(t-1)}-\widetilde{y}_{j t} \leq 0, \quad \forall j \in J, t \in T .
\end{aligned}
$$

Indeed, the objective function (1) ensures that in any optimal solution the startup variables $\widetilde{w}_{j t}$ (resp. $\widetilde{y}_{j t}$ ) take the value 1 only if $g_{j t}-g_{j(t-1)}=1$ (resp. $\left.u_{j t}-u_{j(t-1)}=1\right)$. The resulting model is smaller, but this does not guarantee a better performance. Indeed, the two LP relaxations are identical, and the behavior of the MILP solver is unpredictable. As a matter of fact, for the benchmarks used in our experiments, the smaller model turned out to be equivalent to the larger one for the easy instances, but definitely worse for the difficult ones.

\section{B. Linearizing the Power Production Function}

The performance of a hydro turbine depends on the rate of water discharge and on the net hydraulic head. The value of the net head depends on the water level in the reservoir, the tail-race level and the penstock losses (that are a function of the water flow). It follows that the power generated from a hydro unit is related to the water flow and the reservoir characteristics. For a generic hydro generator unit, the power output $p$ can be expressed as a nonlinear function $\varphi$ of the water flow $q$ and the water volume $v$ in the reservoir, by including the nonlinear relationship that links the net head value to the water volume and the water flow, as well as to the electric loss of the generator, i.e.,

$$
p=\varphi(q, v) .
$$

(Note however that each unit will be characterized by a specific $\varphi$ function.)

Even for a prefixed volume $\widetilde{v}$, the power production, as a function of the water flow, is nonlinear and nonconcave. Net head variation can only be ignored for relatively large reservoirs, in which case power generation is solely dependent on the water flow. (An example of (18) is provided in [5].)

An accurate approximation of $\varphi$ is crucial for modeling the head effect. In [13] the function was approximated by considering a fixed number (three) of water volumes, say $\widetilde{v}^{1}, \widetilde{v}^{2}, \widetilde{v}^{3}$ and interpolating, for each $\widetilde{v}^{r}$, the resulting function

$$
p=\left.\varphi\right|_{\tilde{v}^{r}}(q)
$$

by piecewise linear approximation. To our knowledge, this has been the first successful modeling of the head effect. Indeed, a more accurate approximation of (18) through meshing and triangulation, proposed in [11], proved to be only suitable for small systems (see [12]).

We describe the improvement we propose for approximating (18) in two steps. In the present section we show how to 1) slightly generalize the approach in [13] to a parametric number of water volumes through a classical use of binary variables, 


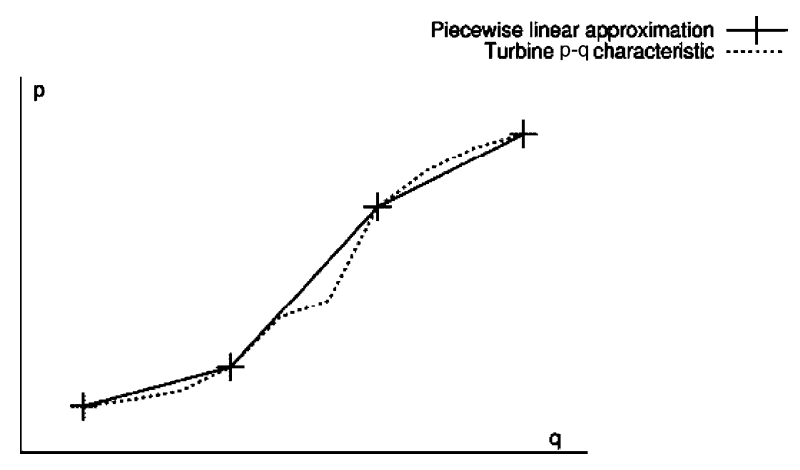

Fig. 1. Simple approximation.

and 2) tighten the linear programming relaxation of the model through a more precise estimation of the upper bound on the power production. The second step, undertaken in the next section, introduces an accurate evaluation of the power production corresponding to intermediate water volumes.

While in [13] the piecewise linear approximation was formulated through the incremental method, we adopted the convex combination method which is mathematically equivalent (see, e.g., [16]) but allows a more intuitive explanation of the enhanced linearization that will be introduced in Section III. We consider $\bar{r}$ volume intervals and $\bar{z}$ coordinates (breakpoints) along the flow axis. Let $R=\{1, \ldots, \bar{r}\}$ and $Z=\{1, \ldots, \bar{z}\}$. Let us introduce the following additional parameters:

$\begin{array}{ll}{\left[H_{r-1}, H_{r}\right) \quad \begin{array}{l}\text { extreme water volumes for interval } r(r \in R) \\ \end{array}} & {\left[\mathrm{m}^{3}\right] ;} \\ Q_{j i} & \text { flow in turbine } j \text { at breakpoint } i(j \in J, \\ & i \in Z)\left[\mathrm{m}^{3} / \mathrm{s}\right] ; \\ P_{j i r} & \text { power from turbine } j \text { at breakpoint } i \text { for } \\ & \text { interval } r(j \in J, i \in Z, r \in R)[\mathrm{MW}] \\ \Delta P_{j r} & \max _{i \in Z}\left\{P_{j i \bar{r}}-P_{j i r}\right\}(j \in J, r \in R)[\mathrm{MW}]\end{array}$

where the last value, introduced for ease of notation, represents the maximum power difference between intervals $r$ and $\bar{r}$. Fig. 1 depicts, for a given water volume, a classical power-flow characteristic of a turbine (dotted line) and its piecewise-linear approximation obtained with four breakpoints (solid line).

Our linearization technique makes use of the following variables: $d_{t r} \quad$ membership status of volume $v_{t}$ wrt interval $r[1$
if $H_{r-1} \leq v_{t}<H_{r}, 0$ otherwise $(t \in T, r \in R)$

$z_{j t i} \quad$ contiguity status of $q_{j t}$ wrt to discretized flow $Q_{j i}$ $\left[1\right.$ if $Q_{j(i-1)}<q_{j t} \leq Q_{j i}$ or $Q_{j i} \leq q_{j t}<Q_{j(i+1)}$, 0 otherwise] $(j \in J, t \in T, i \in Z)$;

$\lambda_{j t i} \quad$ weight of breakpoint $i$ for turbine $j$ in period $t$ $(j \in J, t \in T, i \in Z)$,

where the last variable must obey

$$
0 \leq \lambda_{j t i} \leq 1
$$

The following constraints complete model (1)-(15) by approximating the power production function (18) with a parametric number of water volumes:

$$
\begin{array}{rlrl}
q_{j t}-\sum_{i \in Z} Q_{j i} \lambda_{j t i}-Q_{j}^{-} u_{j t}=0, & & \forall j \in J, t \in T \\
\sum_{i \in Z} \lambda_{j t i}-g_{j t}=0, & & \forall j \in J, t \in T \\
\lambda_{j t i}-z_{j t i} \leq 0, & & \forall j \in J, t \in T, i \in Z \\
z_{j t i}+z_{j t k} \leq 1, & \forall j \in J, t \in T \\
\sum_{r \in R} d_{t r}=1, & \forall t \in T \\
p_{j t}-\sum_{i \in Z} P_{j i r} \lambda_{j t i}-P_{j}^{-} u_{j t}- & & \\
\Delta P_{j r}\left(1-d_{t r}\right) \leq 0, & & \forall j \in J, t \in T, r \in R \\
v_{t}-\sum_{r \in R} H_{r-1} d_{t r} \geq 0, & & \forall t \in T \\
v_{t}-\sum_{r \in R} H_{r} d_{t r} \leq 0, & & \forall t \in T .
\end{array}
$$

Equations (20)-(23) express the water flow $q_{j t}$ of turbine/pump $j$ in period $t$ in the three possible cases seen in Section II. If $u_{j t}=0$, the pump is off and the flow is either zero (if the turbine is off as well, case TP00) or a convex combination of breakpoint flows (case TP10); otherwise the pump is on (so $g_{j t}=0$ from (14)) and there is a constant negative flow $Q_{j}^{-}$(case TP01). Observe indeed that constraints (22)-(23) are inactive when $g_{j t}=0$ (due to (21)), so the first and third case are directly modeled by (20). If instead $g_{j t}=1$ constraints (21) impose that the breakpoint weights sum up to one. Due to constraints (22), any $\lambda_{j t i}$ can only be nonzero if the corresponding binary variable $z_{j t i}$ is one. It follows that constraints (22) and (23) together ensure that: 1) at most two weights can take a positive value, and 2) if this occurs for exactly two weights then they must be contiguous. In summary, the overall effect of constraints (20)-(23) is that $q_{j t}$ is either a constant negative value $Q_{j}^{-}$(case TP01), or null (case TP00), or a piecewise linear approximation (case TP10).

Similarly, (24)-(25) express the power $p_{j t}$ of turbine/pump $j$ in period $t$ for volume interval $r$, in the same three cases. Due to (24), all $d_{t r}$ 's are zero but one. Hence, in the unique volume interval, say $\widetilde{r}$, for which $d_{t \tilde{r}}=1$, the last term of (25) takes the value 0 and (25) itself assumes the same form as (20), but with powers instead of flows. It follows that the same considerations used above ensure that (25) model the three possible cases. The only difference is in the " $\leq$ " sign, which is adopted here, instead of "=". However: 1) this has no effect on the power production, since the objective function (1) ensures the constraint tightness; 2) for all the other volume intervals $r \neq \widetilde{r}$, for which $d_{t r}=0$, the last term of (25) takes the value $\Delta P_{j r}$, thus deactivating the constraint. Finally, (26)-(27) define, for each time period $t$, the two extreme water volumes of the interval where the computed volume $v_{t}$ lies.

Constraints (25) are the crucial difference with respect to the way (18) is approximated in [13]:

1) it is obvious that increasing the number of volume intervals improves the approximation. However, as shown in Section IV, this number cannot be increased too much 
in practice without making the model too big to be handled within reasonable CPU times;

2) constraints (25) could be deactivated (as in [13]) by using the overestimated constant value $\bar{P}_{j}$ instead of $\Delta P_{j r}$. It is known however that adopting tighter values highly strengthens the linear programming relaxation (as confirmed by the computational experiments of Section IV).

\section{ENHANCING THE LINEARIZATION}

In the model of the previous section, for any volume $v_{t}$ belonging, say, to the $r$ th interval $\left[H_{r-1}, H_{r}\right)$, the power production is approximated through a prefixed (static) value $P_{j i r}$ depending on the turbine and the breakpoint (see (25)). The accuracy obtainable in this way heavily depends on the number $\bar{r}+1$ of water volumes $H_{r}$ (corresponding to $\bar{r}$ volume intervals). Such a number, however, cannot be too high without substantially affecting the computational effort. We next show how a good approximation can be obtained by keeping $\bar{r}$ at an effective low value by introducing an enhanced linearization that corrects the estimated power production through two dimensional considerations.

In the enhanced model, for a volume $v_{t}$, belonging, say, to interval $\left[H_{r-1}, H_{r}\right)$, instead of approximating the power production by selecting a point on a single piecewise linear function, we approximate it through a weighted combination of values computed for the two extremes $H_{r-1}$ and $H_{r}$. Let us introduce the power excursion, for turbine $j$ and breakpoint $i$, between intervals $r$ and $r+1$

$$
\Delta P_{j i r}=P_{j i(r+1)}-P_{j i r}(j \in J, i \in Z, r \in R)[\mathrm{MW}]
$$

and the quantity

$$
\begin{aligned}
\overline{\Delta P}_{j i r}= & \max _{k \in Z}\left\{P_{j k(r+1)}-P_{j k r}\right\}-\Delta P_{j i r} \\
& (j \in J, i \in Z, r \in R)[\mathrm{MW}]
\end{aligned}
$$

(with $P_{j i(\bar{r}+1)}=P_{j i \bar{r}}$ ), which is used to deactivate constraints, as will be shown below. Moreover, the enhanced model requires the additional variables

$$
d_{t r}^{\prime}=d_{t r}\left(v_{t}-H_{r-1}\right) /\left(H_{r}-H_{r-1}\right)(t \in T, r \in R)
$$

to represent the weight used for combining the values computed for the two extremes of volume interval $r$.

The enhanced linearized power production function is then computed by (20)-(24), and

$$
\begin{array}{r}
d_{t r}^{\prime}-d_{t r} \leq 0, \quad \forall t \in T, r \in R \\
p_{j t}-P_{j}^{-} u_{j t}-\bar{P}_{j} g_{j t} \leq 0, \quad \forall j \in J, t \in T \\
p_{j t}-\left(\sum_{k \in Z} P_{j k r} \lambda_{j t k}+\Delta P_{j i r} d_{t r}^{\prime}\right)-\left(\Delta P_{j r}\left(1-d_{t r}\right)+\right. \\
\left.\overline{\Delta P}_{j i r}\left(1-z_{j t i}\right)\right) \leq 0, \forall j \in J, t \in T, r \in R, i \in Z \\
v_{t}-\sum_{r \in R}\left(H_{r-1} d_{t r}+\left(H_{r}-H_{r-1}\right) d_{t r}^{\prime}\right)=0, \forall t \in T
\end{array}
$$

which replace (25)-(27).

Equations (28) ensure that, for any time period $t$, the only nonzero weight $d_{t r}^{\prime}$ can occur for the unique interval $r$ for which $d_{t r}=1$ (see (24)). As a consequence, in the summation of (31) the only nonzero term must be equal to the value of $v_{t}$ (given by (3)), thus uniquely determining the corresponding value $d_{t r}^{\prime}=$ $\left(v_{t}-H_{r-1}\right) /\left(H_{r}-H_{r-1}\right)$.
Equations (29) are only active when turbine $j$ is off in period $t$. They thus define the (negative) power consumption due to pump $j$ (case TP01 of Section II), possibly equal to zero if pump $j$ is off as well (case TP00).

Similarly, (30) are only active when turbine $j$ is on in period $t$ (case TP10), since otherwise (29) impose a negative upper bound $P_{j}^{-}$on $p_{j t}$, hence dominating any nonnegative upper bound produced by (30). When active, they determine the (positive) upper bound on the power production, tighter than the one imposed by (29), which is just the variable upper bound $\bar{P}_{j}$. As previously observed, $p_{j t}$ will exactly match such a bound, since the objective function (1) maximizes the power production. Note that in case TP10 the two terms within brackets of (30) play the same role as the second and fourth term of (25), respectively. More precisely, $\Delta P_{j i r} d_{t r}^{\prime}$ imposes a correction to the regular term $\sum_{k \in Z} P_{j k r} \lambda_{j t k}$ while $\overline{\Delta P}_{j i r}\left(1-z_{j t i}\right)$ is used to deactivate those constraints (30) whose breakpoint $i$ is not used (i.e., those for which $z_{j t i}=0$ ).

The correction $\Delta P_{j i r} d_{t r}^{\prime}$ is depicted in Fig. 2. Since $v_{t} \in$ $\left[H_{r-1}, H_{r}\right)$, the power approximation obtained by the model of the previous section would be $P_{j k r} \lambda_{j t k}+P_{j(k+1) r} \lambda_{j t(k+1)}$ (with $\lambda_{j t(k+1)}=1-\lambda_{j t k}$ ), i.e., the value $P^{1}$ in the figure. The enhancement given by (28)-(31) produces a better approximation, namely value $P^{2}$ in the figure. Indeed the correction parameter $d_{t r}^{\prime}$ is computed by considering the relative position of $v_{t}$ within the volume interval $\left[H_{r-1}, H_{r}\right)$ (see (31)). Note that two constraints (30) are active at the same time: the one for $k$ and the one for $k+1$. However, to avoid an overestimation of the correction, the tighter constraint is the one with the smallest $\Delta P_{j i r}$ value. In Fig. 2 such value is $\Delta P_{j k r}$, since the slope of segment $a b$ is smaller than that of segment $c d$.

We illustrate the enhanced linearization technique through a numerical example. Consider Fig. 2, and assume that the $(q, v, p)$ coordinates of the interested points are: $a=(18,1,22)$, $b=(18,5,34), c=(28,1,58)$ and $d=(28,5,98)$. Let us compute the power production corresponding to $q_{j t}=20$ and $v_{t}=2.5$. The linearization of Section II-B would give $P^{1}=22 \lambda_{j t k}+58 \lambda_{j t(k+1)}=29.2$ (by (25) with $\left.\lambda_{j t k}=0.8\right)$. The enhanced linearization gives $P^{2}=P^{1}+(34-22) d_{t r}^{\prime}=33.7$ (by (30) with $\left.d_{t r}^{\prime}=0.375\right)$.

\section{Computational Results}

The models presented in the previous sections were tested by running the MILP solver Ilog-Cplex 10.0 [14] under mathematical programming modeling language AMPL Version 20061102. The tests were executed by sequentially running the code on a single processor of an Intel Core 2 CPU 6600 with $2.40 \mathrm{GHz}$ and $1.94 \mathrm{~GB}$ of RAM. For each instance, a time limit of 7200 seconds was imposed.

Three real-word instances were considered referring to a hydro power plant with one Francis turbine fed by a reservoir of capacity $33 \cdot 10^{6} \mathrm{~m}^{3}$, with a maximum level of $85.25 \mathrm{~m}$. We considered the water inflows and electricity market prices of a week of three different months (namely, April, June and December), selected so as to have considerably different scenarios, with hourly time periods (168 periods per instance). The complete instances are available on line at http://www.or.deis.unibo.it/research_pages/ORinstances/ORinstances.htm. 


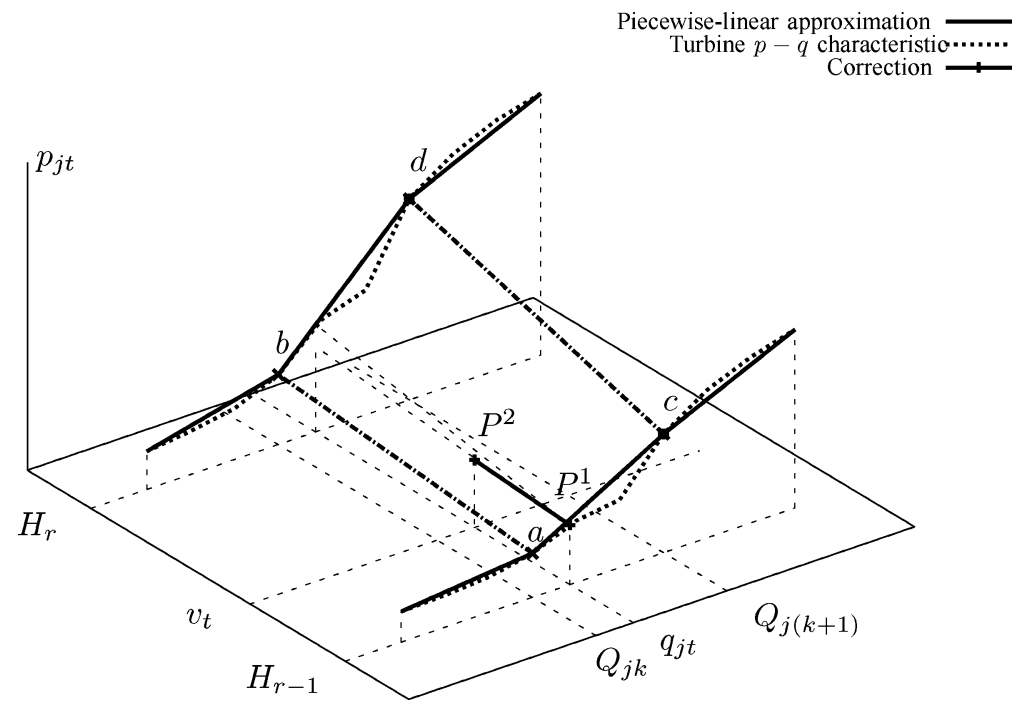

Fig. 2. Enhanced approximation.

We give the results obtained for these instances by three models:

1) basic model, without the improvements introduced in Section II-B ("BDLM-" in the tables), mimicking the model in [13];

2) improved model of Section II-B ("BDLM' in the tables);

3) final enhanced model of Section III ("BDLM+" in the tables). The models were tested with two different $\varphi$ functions, called $\varphi_{1}$ and $\varphi_{2}$, for the turbine (see (18)). Each power production function is approximated by considering three fixed values $H_{i}$ of the water volume. For each water volume, the $p-q$ relationship (19) is represented by a piecewise linear approximation with five breakpoints, as shown in Fig. 3. As already mentioned, in pumping operating mode we assume a constant water flow value (of $0.64 \mathrm{pu}$ ) as well as a constant power consumption (of $0.85 \mathrm{pu}$ ).

The results for $\varphi_{1}$ and $\varphi_{2}$ are given in Tables I and II, respectively. For each instance and model the entries give

a) total number of variables, the number of binary variables, and the number of constraints after Ilog-Cplex preprocessing;

b) value of the initial LP relaxation, obtained by replacing each binary constraint of type $x_{i} \in\{0,1\}$ with $0 \leq x_{i} \leq$ 1

c) value of the improved LP relaxation computed by IlogCplex at the root node through its default cutting plane separation;

d) value of the best solution computed by Ilog-Cplex within the time limit;

e) final percentage gap, computed as

$$
100 \cdot \frac{\text { Best upper bound }- \text { Best solution value }}{\text { Best solution value }}
$$

f) number of branch-and-bound nodes;

g) number of unsolved nodes when the time limit is reached;

h) total CPU time spent.
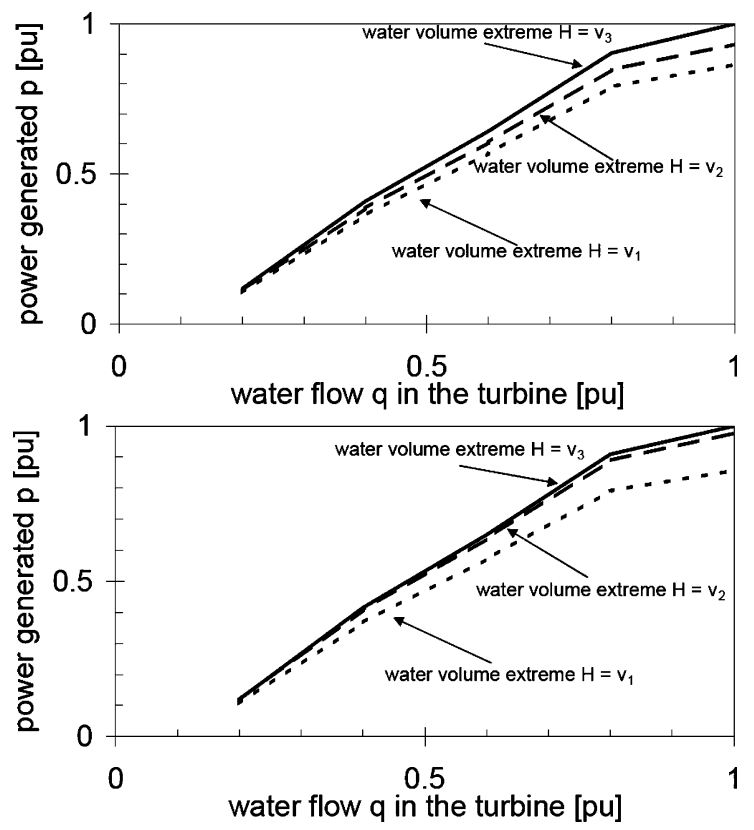

Fig. 3. Piecewise approximation of the relationship (19) for three volume values, namely, $\widetilde{v}^{1}=\underline{V}, \widetilde{v}^{2}=(\underline{V}+\bar{V}) / 2$, and $\widetilde{v}^{3}=\bar{V}$, corresponding to the two different functions considered in the tests: $\varphi_{1}$ (top) and $\varphi_{2}$ (bottom).

The first two lines of each instance refer to two models (BDLM- and BDLM) that provide the same level of approximation of the system (note indeed that the best solution values are identical). By comparing them we can observe that both the initial and the improved LP relaxation of BDLM are much tighter than those of BDLM-. Numbers of nodes and CPU times are generally competitive or much lower for BDLM. Both models produced the optimal solution for all instances within very short CPU times. The difference in the behavior of the two models is mainly due to the use of the tighter $\Delta P_{j r}$ values in (25).

The third line of each instance refer to our most sophisticated model (BDLM+), which provides a more accurate approximation of the real system. This is confirmed by the computational results, which show a considerably better solution value. The higher 
TABLE I

RESUlts FOR A TURBINE WITH THE $\varphi_{1}$ CHARACTERISTIC OF FIG. 3

\begin{tabular}{|c|c|c|c|c|c|c|c|c|c|c|c|}
\hline Instance & Model & $\begin{array}{l}\# \text { of } \\
\text { vars }\end{array}$ & $\begin{array}{r}\text { \# of } \\
\text { bin vars } \\
\end{array}$ & $\begin{array}{r}\# \text { of } \\
\text { const }\end{array}$ & $\begin{array}{r}\text { Initial LP } \\
\text { relaxation value }\end{array}$ & $\begin{array}{l}\text { Improved LP } \\
\text { relaxation value }\end{array}$ & $\begin{array}{r}\text { Best solution } \\
\text { value }\end{array}$ & $\begin{array}{r}\text { Final } \\
\% \text { gap } \\
\end{array}$ & $\begin{array}{r}\text { Number of } \\
\text { nodes }\end{array}$ & $\begin{array}{r}\text { Number of } \\
\text { unsolved nodes }\end{array}$ & \\
\hline \multirow{3}{*}{ April_T168 } & BDLM- & 3,677 & 2,166 & 6,020 & \begin{tabular}{|l|}
$158,802.93$ \\
\end{tabular} & $47,870.30$ & $30,371.44$ & 0.00 & 489 & $\begin{array}{r}0 \\
\end{array}$ & \\
\hline & BDLM & 3,836 & 2,325 & 5,517 & $37,725.41$ & $33,651.66$ & $30,371.44$ & 0.00 & 400 & 0 & \\
\hline & BDLM+ & 4,325 & 2,327 & 8,191 & $95,603.39$ & $46,597.83$ & $42,298.27$ & 0.00 & 3,688 & 0 & \\
\hline \multirow{3}{*}{ June_T168 } & BDLM- & 3,668 & 2,157 & 5,995 & $344,944.69$ & $180,672.99$ & $125,858.46$ & 0.00 & 21,007 & 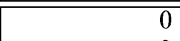 & $\overline{91 .}$. \\
\hline & BDLM & 3,856 & 2,345 & 5,541 & $135,425.69$ & $127,511.18$ & $125,858.46$ & 0.00 & 10,551 & 0 & 29. \\
\hline & BDLM+ & 4,325 & 2,347 & 8,227 & $175,210.15$ & $147,520.10$ & $143,688.22$ & 0.00 & 572,824 & 0 & 2,555 . \\
\hline \multirow{3}{*}{ December _T168 } & BDLM- & 3,693 & 2,182 & 6,045 & $392,563.70$ & $196,000.43$ & $154,702.66$ & 0.00 & 2,336 & 0 & \\
\hline & BDLM & 3,852 & 2,341 & 5,541 & $167,127.86$ & $158,217.74$ & $154,702.66$ & 0.00 & 2,834 & 0 & \\
\hline & BDLM+ & 4,349 & 2,343 & 8,223 & $222,895.80$ & $185,008.07$ & $176,519.74$ & 0.69 & $1,521,821$ & 816,717 & 7,492 . \\
\hline
\end{tabular}

TABLE II

Results For A Turbine With THE $\varphi_{2}$ Characteristic OF Fig. 3

\begin{tabular}{|c|c|c|c|c|c|c|c|c|c|c|c|}
\hline Instance & Model & $\begin{array}{l}\text { \# of } \\
\text { vars }\end{array}$ & $\begin{array}{r}\text { \# of } \\
\text { bin vars } \\
\end{array}$ & $\begin{array}{r}\# \text { of } \\
\text { const }\end{array}$ & $\begin{array}{r}\text { Initial LP } \\
\text { relaxation value }\end{array}$ & $\begin{array}{l}\text { Improved LP } \\
\text { relaxation value }\end{array}$ & $\begin{array}{r}\text { Best solution } \\
\text { value } \\
\end{array}$ & $\begin{array}{r}\text { Final } \\
\% \text { gap } \\
\end{array}$ & $\begin{array}{r}\text { Number of } \\
\text { nodes }\end{array}$ & $\begin{array}{r}\text { Number of } \\
\text { unsolved nodes }\end{array}$ & \\
\hline \multirow{3}{*}{ April_T168 } & BDLM- & 3,677 & 2,166 & 6,020 & $160,254.15$ & $48,111.81$ & $31,098.94$ & 0.00 & 784 & 0 & \\
\hline & BDLM & 3,836 & 2,325 & 5,517 & $38,342.76$ & $35,779.80$ & $31,098.94$ & 0.00 & 687 & 0 & \\
\hline & BDLM+ & 4,325 & 2,327 & 8,191 & $87,151.83$ & $40,533.52$ & $38,347.13$ & 0.00 & 3,890 & 0 & \\
\hline \multirow{3}{*}{ June_T168 } & BDLM- & 3,668 & 2,157 & 5,995 & $347,101.06$ & $180,389.17$ & $132,930.96$ & 0.00 & 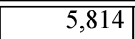 & 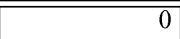 & \\
\hline & BDLM & 3,856 & 2,345 & 5,541 & $136,061.14$ & $133,282.18$ & $132,930.96$ & 0.00 & 2,472 & 0 & \\
\hline & BDLM+ & 4,325 & 2,347 & 8,227 & $162,377.67$ & $136,725.37$ & $135,030.48$ & 0.00 & 11,507 & 0 & \\
\hline \multirow{3}{*}{ December -T168 } & BDLM- & 3,693 & 2,182 & 6,045 & $394,369.19$ & $202,374.20$ & $161,954.09$ & $\overline{0.00}$ & 1,634 & $\overline{00}$ & \\
\hline & BDLM & 3,852 & 2,341 & 5,541 & $170,012.29$ & $165,791.65$ & $161,954.09$ & 0.00 & 2,001 & 0 & \\
\hline & BDLM+ & 4,349 & 2,343 & 8,223 & $208,740.36$ & $172,898.97$ & $169,283.99$ & 0.00 & 20,549 & 0 & 199 \\
\hline
\end{tabular}

TABLE III

Number OF VARIABLES AND CONSTRAINTS FOR THE THREE MODELS CONSIDERING EIGHT CONFIGURATIONS OF $(\bar{t} ; \bar{r} ; \bar{z})$

\begin{tabular}{l|c|c|c|c|c|c|c|c} 
Model & $(24 ; 3 ; 5)$ & $(24 ; 3 ; 8)$ & $(24 ; 5 ; 5)$ & $(24 ; 5 ; 8)$ & $(168 ; 3 ; 5)$ & $(168 ; 3 ; 8)$ & $(168 ; 5 ; 5)$ & $(168 ; 5 ; 8)$ \\
\hline BDLM- & $528 ; 937$ & $672 ; 18,217$ & $528 ; 937$ & $672 ; 18,217$ & 3,$696 ; 6,553$ & 4,$704 ; 127,513$ & 3,$696 ; 6,553$ & 4,$704 ; 127,513$ \\
BDLM & $552 ; 745$ & $696 ; 18,025$ & $600 ; 793$ & $744 ; 18,073$ & 3,$864 ; 5,209$ & 4,$872 ; 126,169$ & 4,$200 ; 5,545$ & 5,$208 ; 126,505$ \\
BDLM+ & $648 ; 1,105$ & $792 ; 18,529$ & $744 ; 1,393$ & $888 ; 18,961$ & 4,$536 ; 7,729$ & 5,$544 ; 129,697$ & 5,$208 ; 9,745$ & 6,$216 ; 132,721$
\end{tabular}

TABLE IV

RESUlts With MORE VOLUME INTERVALS FOR APRIL_T168 AND A TURBINE With THE $\varphi_{1}$ CHARACTERISTIC OF FIG. 3

\begin{tabular}{|c|c|c|c|c|c|c|c|c|c|}
\hline Model & $\begin{array}{r}\text { Numer of } \\
\text { volume intervals }\end{array}$ & $\begin{array}{l}\# \text { of } \\
\text { vars }\end{array}$ & $\begin{array}{r}\text { \# of } \\
\text { bin vars }\end{array}$ & $\begin{array}{l}\text { \# of } \\
\text { const }\end{array}$ & $\begin{array}{r}\text { Best solution } \\
\text { value }\end{array}$ & $\begin{array}{l}\text { Final } \\
\text { \% gap }\end{array}$ & $\begin{array}{r}\text { Number of } \\
\text { nodes }\end{array}$ & $\begin{array}{r}\text { Number of } \\
\text { unsolved nodes }\end{array}$ & $\begin{array}{l}\mathrm{CPU} \\
\text { time }\end{array}$ \\
\hline \multirow{5}{*}{ BDLM } & 3 & 3,836 & 2,325 & 5,517 & $30,371.44$ & 0.00 & 400 & 0 & 3.57 \\
\hline & 4 & 4,011 & 2,500 & 5,709 & $32,985.90$ & 0.00 & 21,411 & 0 & 65.1 \\
\hline & 5 & 4,170 & 2,659 & 5,877 & $34,299.61$ & 0.00 & 30,537 & 0 & 88. \\
\hline & 7 & 4,499 & 2,988 & 6,216 & $34,721.34$ & 4.11 & $2,316,701$ & $1,458,840$ & $7,554$. \\
\hline & 10 & 4,986 & 3,475 & 6,720 & $35,577.54$ & 8.11 & $1,589,801$ & 996,593 & 7,525 \\
\hline BDLM+ & - & 4,325 & 2,327 & 8,191 & 42.298 .27 & 0.00 & 3,688 & 0 & 24. \\
\hline
\end{tabular}

complexity of this model is also reflected by the larger computing times. In spite of this, five instances out of six were solved to optimality. Moreover, for the two "hard" instances of Table I, June_T168 and December_T168, the incumbent solution values after 300 CPU seconds were already 143619.22 and 176508.30 , respectively, i.e., very close to the best solution values.

In Table III we examine the size of the three models by giving the number of variables and constraints in terms of the main parameters, namely $(\bar{t}, \bar{r}$ and $\bar{z})$. The percentage of binary variables was, for all cases, between $50 \%$ and $60 \%$ of the total.

Table IV shows, for instance April_T168 of Table I, how the level of approximation of BDLM improves with the number $\bar{r}$ of volume intervals. The best solution value considerably improves when $\bar{r}$ is increased to four and five, by still requiring acceptable CPU times. Going to higher values (seven and ten) the improvement is marginal, while the increased number of variables and constraints makes the computational effort very heavy, and the model is not solved to optimality within the time limit. In any
TABLE V

RESULTS FOR BDLM+ WITH AND WITHOUT THE BDLM SOLUTION ENFORCED

\begin{tabular}{l|r|r|r} 
Instance & BDLM & $\begin{array}{r}\text { BDLM+ with } \\
\text { BDLM enforced }\end{array}$ & BDLM+ \\
\hline April_T168 & $30,371.44$ & $41,651.54$ & $42,298.27$ \\
\hline June_T168 & $125,858.46$ & $143,384.79$ & $143,688.22$ \\
\hline December_T168 & $154,702.66$ & $175,339.08$ & $176,519.74$
\end{tabular}

case, the best solution value remains far from the one produced by BDLM+ with $\bar{r}=3$ (shown in the last line of the table).

In order to further compare the solutions achieved by models BDLM and BDLM+ we computed the value of the objective function obtained in model BDLM+ when the solution attained by the BDLM model is enforced. We considered a turbine with function $\varphi_{1}$ (see Fig. 3). The three columns of Table V give the value of the solution produced by BDLM, the value produced by BDLM+ if the values of all variables in the BDLM solution (except $p_{j t}$ ) are enforced, and the value produced by BDLM+ from scratch. The results show that the enhanced linearization 
TABLE VI

Results For the MILP Model With SEVEn Volume InTERVALS AND Five BREAKPoINTS

\begin{tabular}{l|l|r|r|r|r|r} 
Instance & $\begin{array}{l}(32)-(33) \\
\text { imposed }\end{array}$ & $\begin{array}{r}\text { Initial LP } \\
\text { relaxation value }\end{array}$ & $\begin{array}{r}\text { Improved LP } \\
\text { relaxation value }\end{array}$ & $\begin{array}{r}\text { Best solution } \\
\text { value }\end{array}$ & $\begin{array}{r}\# \\
\text { nodes }\end{array}$ & $\begin{array}{r}\text { CPU } \\
\text { Time }\end{array}$ \\
\hline $\mathrm{S} 1$ & no & $146,621.07$ & $141,115.26$ & $138,147.38$ & 13,940 & 14.12 \\
$\mathrm{~S} 1$ & yes & $146,621.07$ & $141,097.97$ & $138,144.67$ & 13,403 & 14.99 \\
$\mathrm{~S} 1 \mathrm{~A}$ & yes & $146,621.07$ & $141,002.27$ & $137,540.30$ & $2,535,628$ & $2,507.13$ \\
\hline \hline $\mathrm{S2}$ & no & $158,510.03$ & $152,301.95$ & $134,148.57$ & 64,798 & 76.07 \\
$\mathrm{~S} 2$ & yes & $158,510.03$ & $151,532.19$ & $134,148.57$ & 66,553 & 74.85 \\
$\mathrm{~S} 2 \mathrm{~A}$ & yes & $158,510.03$ & $152,117.09$ & $134,141.57$ & 57,645 & 72.10 \\
\hline \hline $\mathrm{S3}$ & no & $251,528.59$ & $229,658.19$ & $199,610.76$ & 4,428 & 8.76 \\
$\mathrm{~S} 3$ & yes & $251,528.59$ & $230,124.38$ & $199,610.76$ & 6,381 & 13.73 \\
$\mathrm{~S} 3 \mathrm{~A}$ & yes & $251,528.59$ & $229,359.16$ & $199,277.13$ & 3,456 & 8.58
\end{tabular}

of BDLM+ allows the MILP solver to find truly improved solutions wrt BDLM, i.e., the different values in the tables do not merely correspond to different measures.

Other authors considered real cases in which the turbines have forbidden operating zones. For example, [4] considers one multiplant daily instance ( 24 time periods) in which each plant has a single forbidden flow interval. In order to test our model on such situations, we extracted from such instance three single plant instances (named S1, S2 and S3), and added to the BDLM+ model specific constraints to forbid a flow interval, namely

$$
\begin{aligned}
q_{j t}-\underline{F}_{j}-\bar{Q}_{j}\left(1-x_{j t}\right) \leq 0, & \forall j \in J, t \in T \\
q_{j t}-\bar{F}_{j}\left(1-x_{j t}\right)-Q_{j}^{-} x_{j t} \geq 0, & \forall j \in J, t \in T
\end{aligned}
$$

where $\left[\underline{F}_{j}, \bar{F}_{j}\right]$ is the prohibited operating zone for turbine $j$, and $x_{j t}$ is a binary variable that takes the value 1 when $Q_{j}^{-} \leq$ $q_{j t} \leq \underline{F}_{j}$ or the value 0 when $\bar{F}_{j} \leq q_{j t} \leq \bar{Q}_{j}$. Note that (32) (resp. (33)) is not active when $x_{j t}=0$ (resp. $x_{j t}=1$ ). The outcome of the experiments is reported in Table VI, where the second column tells whether constraints (32)-(33) are added to BDLM+. The first two lines of each instance give the results obtained by solving such instances with BDLM+, with and without the new constraints. The optimal solutions only slightly differ on instance S1, thus showing that (32)-(33) were (almost) not active. In order to better test the model, we artificially moved the forbidden intervals to optimal regions, thus creating more challenging instances S1A, S2A and S3A. The behavior of BDLM+ with (32)-(33), given in the third line of each instance, results to be still satisfactory.

In order to illustrate the differences between the two proposed models, BDLM and BDLM+, Figs. 4-7 show the scheduling results they produce for instance June_T168 with power production function $\varphi_{1}$.

Fig. 4 shows the water volume values in the basin, taking into account the minor amount of natural inflow (just fractions of $\mathrm{m}^{3} / \mathrm{s}$ ) and the different volume values at the beginning and at the end of the week, imposed by the considered instance (namely $32.5 \cdot 10^{6} \mathrm{~m}^{3}$ and $26.5 \cdot 10^{6} \mathrm{~m}^{3}$, respectively). The horizontal lines show the three volume intervals adopted for linearizing the power production function. As already mentioned, the inflow and the initial and the final values were taken from the real world data of a power plant. Fig. 5 compares the calculated profiles of the water flows discharged or pumped by the hydro unit during the considered week.

Fig. 6 compares the power production levels, also giving the considered market price profile during the week, while

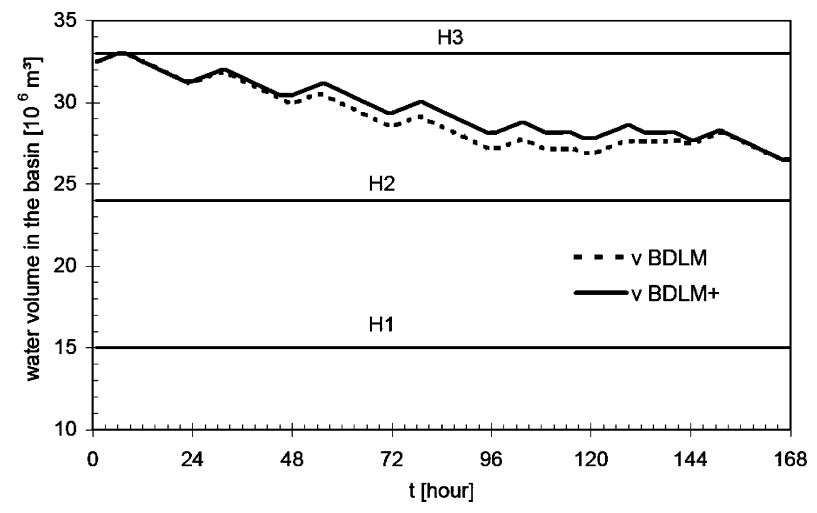

Fig. 4. Water volumes.

Fig. 7 compares the curves of the accumulated profits in the two models. Fig. 6 shows that the maximum output of the unit calculated by BDLM+ is larger than that obtained by BDLM, due to the more refined representation of the head effect in function $\varphi$, that results in a higher profile of the calculated water volumes in the basin. This explains 1) the larger profit levels estimated by BDLM+, as shown in Fig. 7, and 2) the different scheduling, mainly for the second last day (Sunday), characterized by lower market prices. (The considered week starts on Monday midnight and runs through the next Monday midnight.) For Sunday morning, BDLM suggests not to produce, due to the low value of the water volume stored in the basin (which should be saved in order to be available at the more profitable market price levels of the following day), whilst $\mathrm{BDLM}+$ recommends to produce, allowing therefore a superior exploitation of the natural resource.

\section{CONCLUSIONS}

We have considered the problem of determining the commitment and the power generation of a single reservoir pumpstorage hydro power plant. Starting from the MILP model proposed in [13], we have obtained an enhanced model that takes into account relevant technological aspects, such as ramp transitions, pump-storage and head effect. In particular, we have proposed a sophisticated approximation of the head effect in which the linearization is enhanced through two dimensional considerations. The proposed MILP model allows to accurately represent most of the hydroelectric system characteristics, and turns out to be computationally solvable for a planning horizon of one week, proving the high efficiency of modern MILP software tools, both in terms of solution accuracy and computing time. 


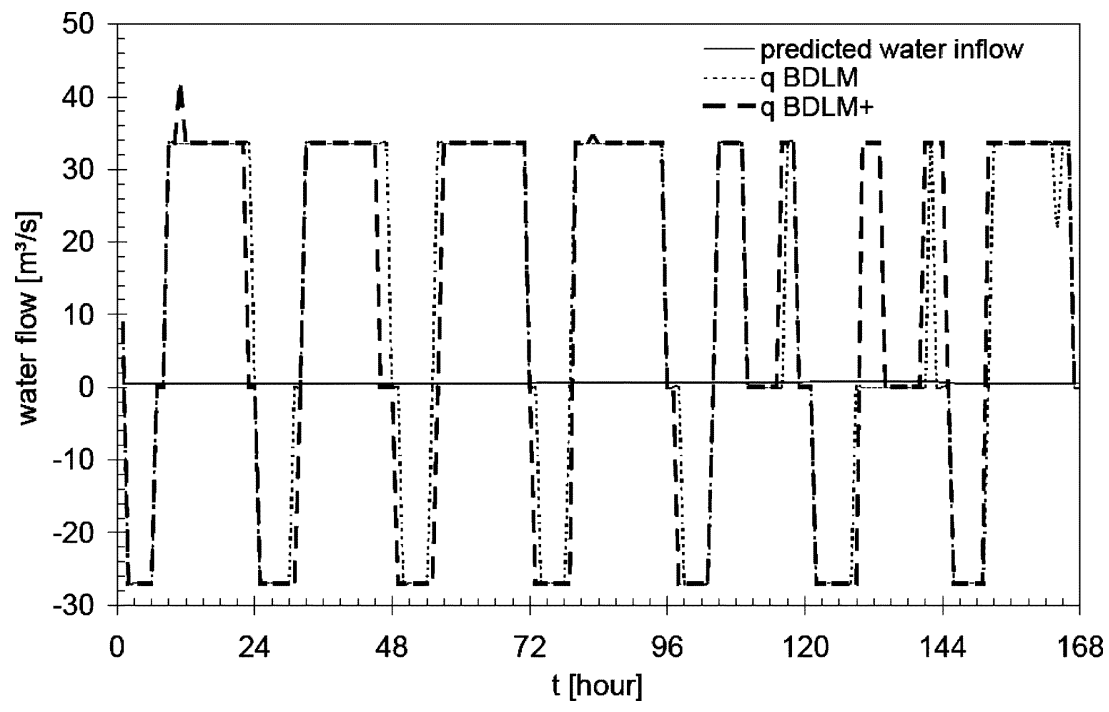

Fig. 5. Inflow and flows.

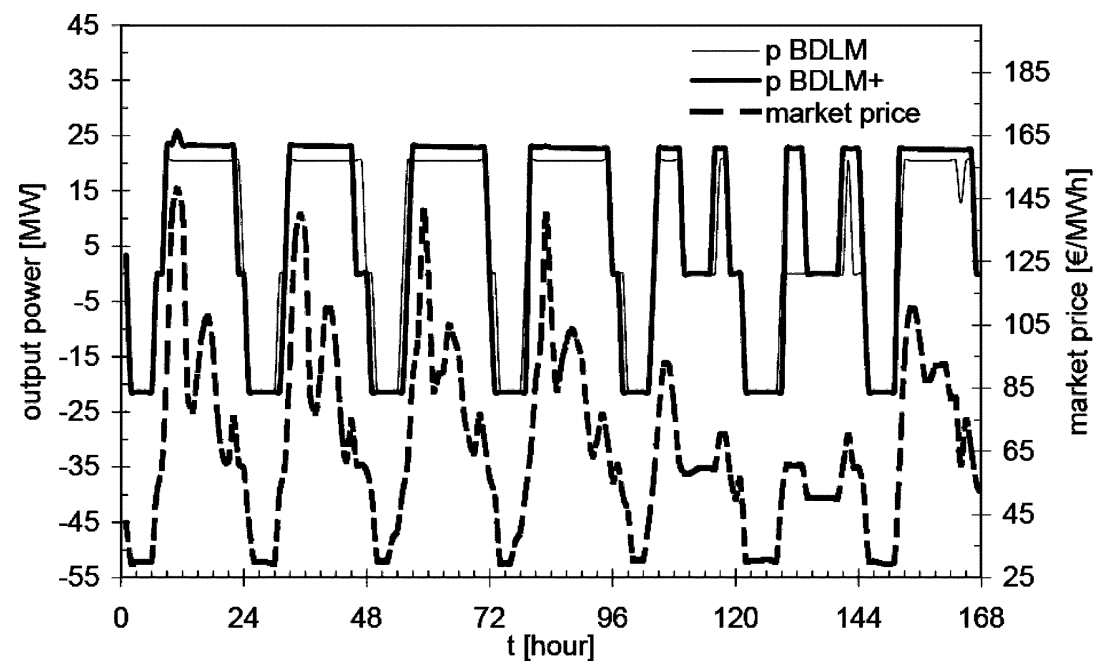

Fig. 6. Price and powers.

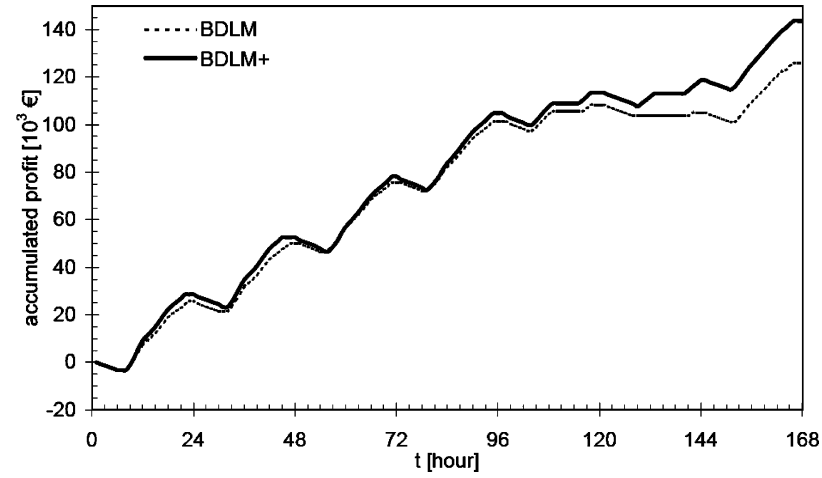

Fig. 7. Profit.

Future developments could involve the extension of the model to represent hydrological interdependent plants in cascade hydro systems. This task is far from being trivial for various reasons. It is clear that the model would require the continuity equations of the hydro reservoirs, taking into account the relevant constraints such as branch flow limits and water travel time (see, e.g., [7]-[9], [11], [13]). The main drawback, however, could be that a cascade hydro system model for, say, $k$ power plants would multiply by $k$ the number of variables and constraints of BDLM+, and the computational experiments of Section IV have shown that the performance of the model is heavily affected by its size. In addition, the sophisticated linear approximations introduced to model the nonlinear aspects could deteriorate the accuracy of a much larger model. In our opinion the extension to the modeling of schemes with a combination of multiple reservoirs, rivers, weirs and hydroelectric plants in series and parallel combinations could require a different use of the MILP solver, which should not be run as a black box, but embedded in a more involved algorithmic framework.

\section{ACKNOWLEDGMENT}

The authors would like to thank Prof. C. Alberto Nucci for his helpful comments. The authors would also like to thank anonymous referees for useful comments that improved the presentation. 


\section{REFERENCES}

[1] N. Padhy, "Unit commitment-A bibliographical survey," IEEE Trans. Power Syst., vol. 19, no. 2, pp. 1196-1205, May 2004.

[2] M. Piekutowki, T. Litwinowcz, and R. Frowd, "Optimal short-term scheduling for a large-scale cascaded hydro system," IEEE Trans. Power Syst., vol. 9, no. 2, pp. 805-811, May 1994.

[3] J. Catalão, S. Mariano, V. Mendes, and L. Ferreira, "Parameterisation effect on the behaviour of a head-dependent hydro chain using a nonlinear model," Elect. Power Syst. Res., vol. 76, pp. 404-412, 2006.

[4] N. Sinha, R. Chakrabarti, and P. Chattopadhyay, "Fast evolutionary programming techniques for short-term hydrothermal scheduling," IEEE Trans. Power Syst., vol. 18, no. 1, pp. 214-220, Feb. 2003.

[5] S. Orero and M. Irving, "A genetic algorithm modelling framework and solution technique for short term optimal hydrothermal scheduling," IEEE Trans. Power Syst., vol. 13, no. 2, pp. 501-518, May 1998.

[6] N. Lu, J. Chow, and A. Desrochers, "Pumped-storage hydro-turbine bidding strategies in a competitive electricity market," IEEE Trans. Power Syst., vol. 19, no. 2, pp. 834-841, May 2004.

[7] E. Finardi, E. Da Silva, and C. Sagastizabal, "Solving the unit commitment problem of hydropower plants via Lagrangian relaxation and sequential quadratic programming," Comput. Appl. Math., vol. 24, no. 3, pp. 317-341, 2005.

[8] C. Chang and J. Waight, "A mixed integer linear programming based hydro unit commitment," in Proc. Power Eng. Soc. Summer Meeting, Jul. 18-22, 1999.

[9] G. Chang, M. Aganagic, J. Waight, J. Medina, T. Burton, S. Reeves, and M. Christoforidis, "Experiences with mixed integer linear programming based approaches on short-term hydro scheduling," IEEE Trans. Power Syst., vol. 16, no. 4, pp. 743-749, Nov. 2001.

[10] A. Baillo, M. Ventosa, A. Ramos, M. Rivier, and A. Canseco, "Strategic unit commitment for generation companies in deregulated electricity markets," in Proc. 1999 DIMACS/EPRI Workshop, 1999.

[11] J. García-González and G. Castro, "Short-term hydro scheduling with cascaded and head-dependent reservoirs based on mixed-integer linear programming," in IEEE Porto Power Tech, Sep. 10-13, 2001.

[12] J. García-González, E. Parrilla, and A. Mateo, "Risk-averse profit-based optimal scheduling of a hydro-chain in the day-ahead electricity market," Eur. J. Oper. Res., vol. 181, no. 3, pp. 1354-1369, 2007.

[13] A. Conejo, J. Arroyo, J. Contreras, and F. Villamor, "Self-scheduling of a hydro producer in a pool-based electricity market," IEEE Trans. Power Syst., vol. 17, no. 4, pp. 1265-1272, Nov. 2002.

[14] Ilog CPLEX. [Online]. Available: http://www.ilog.com/products/cplex.

[15] M. Carrión and J. M. Arroyo, "A computationally efficient mixed-integer linear formulation for the thermal unit commitment problem," IEEE Trans. Power Syst., vol. 21, no. 3, pp. 1371-1378, Aug. 2006.

[16] A. Keha, I. de Farias, and G. Nemhauser, "Models for representing piecewise linear cost functions," Oper. Res. Lett., vol. 32, pp. 44-48, 2004.

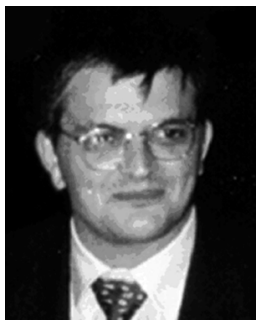

tion scheduling.
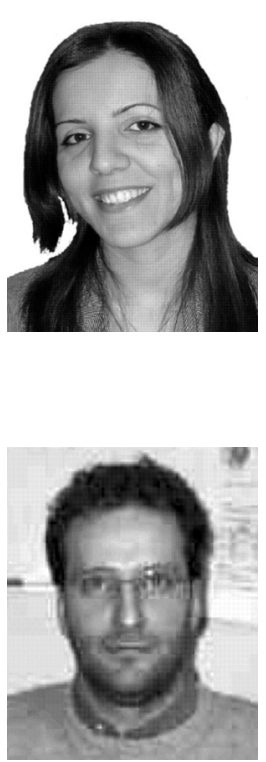

Andrea Lodi was born in Bologna, Italy, in 1969.

$\mathrm{He}$ is a Professor of operations research at the University of Bologna. His main research interests are in the fields of integer programming and combinatorial optimization. He is author of more than 50 papers published in international journals and books.

Prof. Lodi is Associate Editor of Mathematical Programming Series A and INFORMS Journal on Computing.

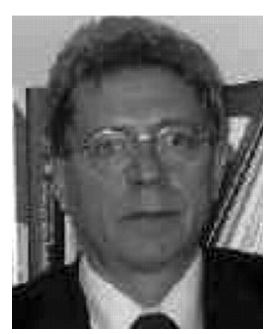

Silvano Martello was born in Bologna, Italy, in 1948.

$\mathrm{He}$ is a Professor of operations research at the University of Bologna. His main research fields are combinatorial optimization and mathematical programming. He is author with $\mathrm{P}$. Toth of the book Knapsack Problems: Algorithms and Computer Implementations (New York: Wiley, 1990). He is author of more than 120 papers.

Prof. Martello is the Editor-in-Chief of $4 O R-A$ Quarterly Journal of Operations Research. 